Department of Mathematics

A dual consistent summation-by-parts formulation for the linearized incompressible Navier-Stokes equations posed on deforming domains

Samira Nikkar and Jan Nordström

LiTH-MAT-R--2016/10--SE 
Department of Mathematics

Linköping University

S-581 83 Linköping 


\title{
A dual consistent summation-by-parts formulation for the linearized incompressible Navier-Stokes equations posed on deforming domains
}

\author{
Samira Nikkara ${ }^{\mathrm{a}}$,Jan Nordström ${ }^{\mathrm{b}}$ \\ ${ }^{a}$ Department of Mathematics, Computational Mathematics, Linköping University, \\ SE-581 83 Linköping, Sweden (samira.nikkar@liu.se). \\ ${ }^{b}$ Department of Mathematics, Computational Mathematics, Linköping University, \\ SE-58183 Linköping, Sweden (jan.nordstrom@liu.se).
}

\begin{abstract}
In this article, well-posedness and dual consistency of the linearized incompressible Navier-Stokes equations posed on time-dependent spatial domains are studied. To simplify the derivation of the dual problem, the second order formulation is transformed to first order form. Boundary conditions that simultaneously lead to well-posedness of the primal and dual problems are derived.

We construct fully discrete finite difference schemes on summation-byparts form, in combination with the simultaneous approximation technique. We prove energy stability and discrete dual consistency. Moreover, we show how to construct the penalty operators such that the scheme automatically adjusts to the variations of the spatial domain, and as a result, stability and discrete dual consistency follow simultaneously.

The method is illustrated by considering a deforming time-dependent spatial domain in two dimensions. The numerical calculations are performed using high order operators in space and time. The results corroborate the stability of the scheme and the accuracy of the solution. We also show that linear functionals are superconverging. Additionally, we investigate the convergence of non-linear functionals and the divergence of the solution.
\end{abstract}

Keywords: Incompressible Navier-Stokes equations, Deforming domain, Stability, Dual consistency, High order accuracy, Superconvergence 


\section{Introduction}

In many applications, functionals of the solution of an Initial Boundary Value Problem (IBVP) are more interesting than the solution itself. These functionals are weighted integrals of the solution over the spatial domain. Typical examples are the lift and drag coefficients in aerodynamics applications. One of the most interesting result of having a dual consistent finite difference approximation is that it leads to superconverging linear functionals $[9,10]$.

Dual consistent schemes on Summation-by-Parts (SBP) form in combination with the Simultaneous Approximation Term (SAT) technique, are investigated in $[6,7,8,9,10]$ for a variety of problems posed on fixed spatial domains. It was found that the only requirement for having a dual consistent scheme on SBP-SAT form is that a specific subset of values for the SAT penalty coefficients/operators among a range of values for which stability is guaranteed must be chosen. Consequently, superconverging linear functional approximations comes with no additional computational costs.

In this article, we extend the SBP-SAT dual consistency approach to IBVPs posed on time-dependent spatial domains. We consider the twodimensional linearized incompressible Navier-Stokes equations, and start by reducing the problem to a first order system. Next, a time-dependent coordinate transformation is used to map the problem from a moving into a fixed spatial domains. Additionally, we apply the techniques in [4] such that the Numerical Geometric Conservation Law (NGCL) is preserved under the coordinate transformation.

The rest of this article proceeds as follows. In section 2, we analyze the continuous primal problem, choose well-posed boundary conditions and derive the adjoint problem together with well-posed dual boundary conditions. In section 3, the discrete problem is constructed, stability of the primal and dual problems are investigated and the requirements for a dual consistent approximation are specified. Numerical experiments are performed in section 4 , where we show the convergence behavior of the solution, divergence and functionals. Finally, we summarize and draw conclusions in section 5 . 


\section{The incompressible Navier-Stokes equations}

Consider the two-dimensional incompressible Navier-Stokes equations

$$
\begin{aligned}
u_{t}+u u_{x}+v u_{y}+p_{x} & =\mu\left(u_{x x}+u_{y y}\right), \\
v_{t}+u v_{x}+v v_{y}+p_{y} & =\mu\left(v_{x x}+v_{y y}\right), \\
u_{x}+v_{y} & =0,
\end{aligned}
$$

where $(x, y) \in \Omega(t)$ and $t \in[0, T]$. In (1), $x, y$ and $t$ are the spatial coordinates and time, respectively. The subscripts $t, x$ and $y$ denote partial derivatives in their respective directions, $p$ is the pressure, $\mu>0$ is the constant viscosity and $\Omega(t)$ is a moving and/or deforming spatial domain.

We rewrite (1) in matrix vector form as

$$
S U_{t}+\hat{A}(U) U_{x}+\hat{B}(U) U_{y}=C\left(U_{x x}+U_{y y}\right),
$$

where $U=[u, v, p]^{T}$,

$$
S=\left[\begin{array}{lll}
1 & 0 & 0 \\
0 & 1 & 0 \\
0 & 0 & 0
\end{array}\right], \hat{A}(U)=\left[\begin{array}{lll}
u & 0 & 1 \\
0 & u & 0 \\
1 & 0 & 0
\end{array}\right], \hat{B}(U)=\left[\begin{array}{lll}
v & 0 & 0 \\
0 & v & 1 \\
0 & 1 & 0
\end{array}\right],
$$

and $C=\mu S$. We linearize (2) and obtain the constant coefficient problem as

$$
S U_{t}+A U_{x}+B U_{y}=C\left(U_{x x}+U_{y y}\right) .
$$

In (4), $A=\hat{A}(\bar{U})$ and $B=\hat{B}(\bar{U})$ where the bar sign indicates the reference state around which we have linearized.

In order to simplify the derivation of the dual problem, the problem (4) is reduced to a first order system, through the transformations $V=U_{x}$ and $W=U_{y}$. The first order formulation also makes it possible to achieve design order of acuracy of the divergence. The result is

$$
\mathbf{S U}_{t}+\mathbf{A U}_{x}+\mathbf{B U}_{y}+\mathbf{C U}=0,
$$

where $\mathbf{U}=\left[U^{T}, V^{T}, W^{T}\right]^{T}$ and

$$
\mathbf{S}=\left[\begin{array}{ccc}
S & & \\
& 0 & \\
& & 0
\end{array}\right], \mathbf{A}=\left[\begin{array}{ccc}
A & -C & 0 \\
-C & 0 & 0 \\
0 & 0 & 0
\end{array}\right], \mathbf{B}=\left[\begin{array}{ccc}
B & 0 & -C \\
0 & 0 & 0 \\
-C & 0 & 0
\end{array}\right], \mathbf{C}=\left[\begin{array}{lll}
0 & 0 & 0 \\
0 & C & 0 \\
0 & 0 & C
\end{array}\right] .
$$

Remark 1. Note that all the systems (2), (4) and (6) are symmetric. 


\subsection{Time-dependent coordinate transformation}

An invertible time-dependent coordinate transformation is considered,

$$
\begin{aligned}
& x=x(\tau, \xi, \eta), \quad y=y(\tau, \xi, \eta), \quad t=\tau, \\
& \xi=\xi(t, x, y), \quad \eta=\eta(x, y, t), \quad \tau=t .
\end{aligned}
$$

The transformation satisfies

$$
\left[\begin{array}{l}
\partial / \partial \xi \\
\partial / \partial \eta \\
\partial / \partial \tau
\end{array}\right]=\underbrace{\left[\begin{array}{lll}
x_{\xi} & y_{\xi} & 0 \\
x_{\eta} & y_{\eta} & 0 \\
x_{\tau} & y_{\tau} & 1
\end{array}\right]}_{:=[J]}\left[\begin{array}{l}
\partial / \partial x \\
\partial / \partial y \\
\partial / \partial t
\end{array}\right],
$$

where the subscripts $\tau, \xi$ and $\eta$ denote partial derivatives and $[J]$ is the Jacobian matrix of the transformation. The metric relations [11, 23] are

$$
\begin{array}{lll}
J \xi_{t}=x_{\eta} y_{\tau}-x_{\tau} y_{\eta}, & J \xi_{x}=y_{\eta}, & J \xi_{y}=-x_{\eta}, \\
J \eta_{t}=y_{\xi} x_{\tau}-x_{\xi} y_{\tau}, & J \eta_{x}=-y_{\xi}, & J \eta_{y}=x_{\xi},
\end{array}
$$

where $J=x_{\eta} y_{\xi}-x_{\xi} y_{\eta}>0$ is the determinant of $[J]$.

All non-singular transformations satisfy the Geometric Conservation Law (GCL)

$$
\begin{aligned}
& J_{\tau}+\left(J \xi_{t}\right)_{\xi}+\left(J \eta_{t}\right)_{\eta}=0 \\
&\left(J \xi_{x}\right)_{\xi}+\left(J \eta_{x}\right)_{\eta}=0 \\
&\left(J \xi_{y}\right)_{\xi}+\left(J \eta_{y}\right)_{\eta}=0 .
\end{aligned}
$$

Now that the transformation is applied to the spatial domain $\Omega(t)$ and results in a fixed domain, $\Phi$. A schematic of $\Omega(t)$ and $\Phi$ is given in Figure 1 .

The chain rule applied to (5) and the result multiplied with $J$ yields

$$
\mathcal{S} \mathbf{U}_{\tau}+\mathcal{A} \mathbf{U}_{\xi}+\mathcal{B} \mathbf{U}_{\eta}+\mathcal{C} \mathbf{U}=0, \quad(\xi, \eta) \in \Phi, \quad \tau \in[0, T],
$$

where

$$
\begin{aligned}
\mathcal{S} & =J \mathbf{S} \\
\mathcal{A} & =\left(J \xi_{t}\right) \mathbf{S}+\left(J \xi_{x}\right) \mathbf{A}+\left(J \xi_{y}\right) \mathbf{B} \\
\mathcal{B} & =\left(J \eta_{t}\right) \mathbf{S}+\left(J \eta_{x}\right) \mathbf{A}+\left(J \eta_{y}\right) \mathbf{B} \\
\mathcal{C} & =J \mathbf{C} .
\end{aligned}
$$

Finally, the GCL given in (10) is applied to (12) and results in

$$
\mathcal{S}_{\tau}+\mathcal{A}_{\xi}+\mathcal{B}_{\eta}=0
$$

by which (11) can be rewritten in conservative form, as

$$
(\mathcal{S} \mathbf{U})_{\tau}+(\mathcal{A U})_{\xi}+(\mathcal{B} \mathbf{U})_{\eta}+\mathcal{C} \mathbf{U}=0, \quad(\xi, \eta) \in \Phi, \quad \tau \in[0, T] .
$$




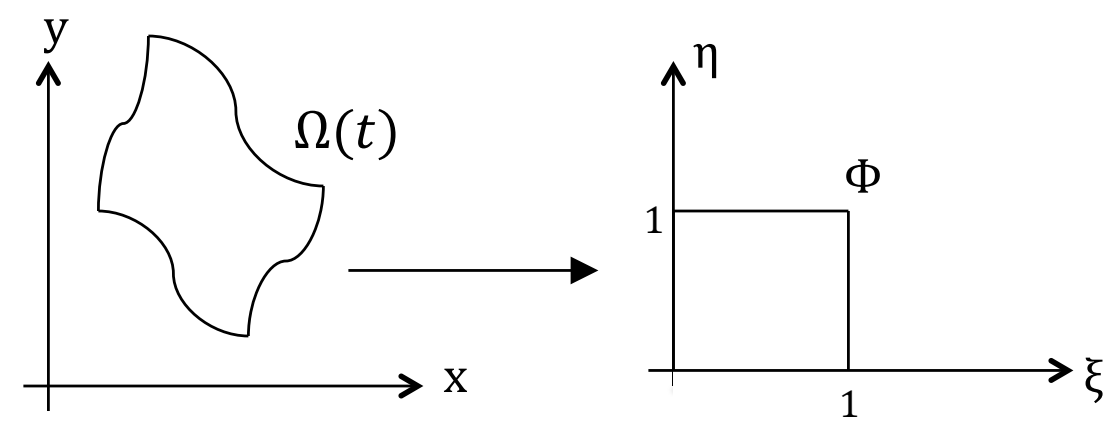

Figure 1: A schematic of the domain in Cartesian and curvilinear coordinates

Remark 2. The equation (14) is denoted as conservative with a slight abuse of notation, since it has a lower order term that comes from the reduction to a first order system.

\subsection{Well-posedness of the primal problem}

The energy method (multiplying (14) from the left with the transpose of the solution and integrating over the spatial and temporal domains) gives

$$
\begin{aligned}
\|\mathbf{U}(T, \xi, \eta)\|_{\mathcal{S}(T)}^{2}-\|\mathbf{U}(0, \xi, \eta)\|_{\mathcal{S}(0)}^{2} & +2 \int_{0}^{T} \iint_{\Phi} \mathbf{U}^{T} \mathcal{C} \mathbf{U} d \Phi d \tau= \\
& -\int_{0}^{T} \oint_{\delta \Phi} \mathbf{U}^{T} D \mathbf{U} d s d \tau .
\end{aligned}
$$

In (15), $D=(\mathcal{A}, \mathcal{B}) \cdot n=n_{1} \mathcal{A}+n_{2} \mathcal{B}$, where $n=\left(n_{1}, n_{2}\right)$ is the outward pointing unit normal from the boundary of $\Phi$, denoted by $\delta \Phi$. Additionally, the norms are defined by

$$
\|\mathbf{U}\|_{\mathcal{S}}^{2}=\iint_{\Phi} \mathbf{U}^{T} \mathcal{S} \mathbf{U} d \xi d \eta=\iint_{\Phi} U^{T} J S U d \xi d \eta=\iint_{\Phi} J\left(u^{2}+v^{2}\right) d \xi d \eta .
$$

The volume term in (15) is dissipative, i.e. matrix $\mathcal{C} \geq 0$.

The matrix $D$ in (15) can be diagonalized as $D=X \Lambda X^{T}$, where the decomposition $\Lambda=\Lambda_{+}+\Lambda_{-}$splits up the eigenvalue matrix into non-negative and negative parts, respectively. Moreover, the eigenvector matrix $X$ can 
be rearranged as $X=\left[X_{+}, X_{-}\right]$where $X_{+}$and $X_{-}$are the eigenvectors corresponding to $\Lambda_{+}$and $\Lambda_{-}$, respectively. We can now rewrite (15) as

$$
\begin{aligned}
& \|\mathbf{U}(T, \xi, \eta)\|_{\mathcal{S}(T)}^{2}-\|\mathbf{U}(0, \xi, \eta)\|_{\mathcal{S}(0)}^{2}+2 \int_{0}^{T} \iint_{\Phi} \mathbf{U}^{T} \mathcal{C} \mathbf{U} d \Phi d \tau= \\
& -\int_{0}^{T} \oint_{\delta \Phi}\left(X_{+}^{T} \mathbf{U}\right)^{T} \Lambda_{+}\left(X_{+}^{T} \mathbf{U}\right) d s d \tau-\int_{0}^{T} \oint_{\delta \Phi}\left(X_{-}^{T} \mathbf{U}\right)^{T} \Lambda_{-}\left(X_{-}^{T} \mathbf{U}\right) d s d \tau .
\end{aligned}
$$

In order to bound the energy of the solution in (17), boundary conditions must be applied to the potentially growing terms, associated with the negative eigenvalues. For simplicity, we choose the characteristic boundary conditions

$$
\left(X_{-}^{T} \mathbf{U}\right)_{j}=\left(X_{-}^{T} \mathbf{U}_{\infty}\right)_{j} \text { if } \Lambda_{j j}<0
$$

where $j \in\{1,2, \ldots, 9\}$ and $\mathbf{U}_{\infty}$ is a reference solution at the boundary $\delta \Phi$. Moreover, we consider an initial condition of the form $\mathbf{U}(0, \xi, \eta)=f(\xi, \eta)$.

Strong imposition of the initial and boundary conditions to (17) leads to the energy estimate,

$$
\begin{array}{r}
\|\mathbf{U}(T, \xi, \eta)\|_{\mathcal{S}(T)}^{2}+\int_{0}^{T} \oint_{\delta \Phi}\left(X_{+}^{T} \mathbf{U}\right)^{T} \Lambda_{+}\left(X_{+}^{T} \mathbf{U}\right) d s d \tau+2 \int_{0}^{T} \int_{\Phi}^{\mathbf{U}^{T} \mathcal{C} \mathbf{U} d \Phi d \tau} \\
=\|f(\xi, \eta)\|_{\mathcal{S}(0)}^{2}-\int_{0}^{T} \oint_{\delta \Phi}\left(X_{-}^{T} \mathbf{U}_{\infty}\right)^{T} \Lambda_{-}\left(X_{-}^{T} \mathbf{U}_{\infty}\right) d s d \tau
\end{array}
$$

The estimate (19) guarantees uniqueness of the solution and existence is given by the fact that we use the correct (minimal) number of boundary conditions equal to the number of elements in $\Lambda_{-}$. We can summarize the results of this section in the following proposition.

Proposition 1. Equation (14) augmented with the boundary condition (18) is strongly well-posed and has the bound in (19).

For later reference, consider the south boundary where $\eta=0$ (indicated by subscript $s$ ) and $n=(-1,0)$. Then, $D_{s}=-\mathcal{B}_{s}=-X_{\mathcal{B}_{s}} \Lambda_{\mathcal{B}_{s}} X_{\mathcal{B}_{s}}^{T}$, and the estimate in (19) becomes 


$$
\begin{aligned}
& \|\mathbf{U}(T, \xi, \eta)\|_{\mathcal{S}(T)}^{2}-\int_{0}^{T} \int\left[\left(X_{\mathcal{B}_{s}}\right)_{-}^{T} \mathbf{U}\right]^{T}\left(\Lambda_{\mathcal{B}_{s}}\right)_{-}\left[\left(X_{\mathcal{B}_{s}}\right)_{-}^{T} \mathbf{U}\right] d \xi d \tau+2 \int_{0}^{T} \int_{\Phi} \mathbf{U}^{T} \mathcal{C} \mathbf{U} d \Phi d \tau \\
& =\|f(\xi, \eta)\|_{\mathcal{S}(0)}^{2}+\int_{0}^{T} \int\left[\left(X_{\mathcal{B}_{s}}\right)_{+}^{T} \mathbf{U}_{\infty}\right]^{T}\left(\Lambda_{\mathcal{B}_{s}}\right)_{+}\left[\left(X_{\mathcal{B}_{s}}\right)_{+}^{T} \mathbf{U}_{\infty}\right] d \xi d \tau
\end{aligned}
$$

\subsection{The dual problem}

To derive the dual problem, we consider (14) augmented with a functional, homogeneous initial and boundary conditions and a forcing function $F$, as

$$
\begin{array}{rlrl}
(\mathcal{S U})_{\tau}+(\mathcal{A U})_{\xi}+(\mathcal{B U})_{\eta}+\mathcal{C} \mathbf{U} & =F, & (\xi, \eta) & \in \Phi, \\
X_{-}^{T} \mathbf{U} & =0, & (\xi, \eta) \in \delta \Phi, \\
U(\xi, \eta) & =0, & & =0 \\
\mathcal{J}(\mathbf{U}) & =(\mathbf{U}, G) . &
\end{array}
$$

In actual calculations, the right hand side $F$ may be identically zero. Here we use it to derive the dual problem. In $(21), \mathcal{J}(\mathbf{U})$ is a linear functional of the solution with a weight function $G$ given by

$$
\mathcal{J}(\mathbf{U})=(\mathbf{U}, G):=\iint_{\Phi} \mathbf{U}^{T} G d \xi d \eta .
$$

Remark 3. In the remainder of the analysis, is is convenient to switch between the integral and inner product notations in (22).

Our objective is to find the dual solution $\Theta=[\theta, \Psi, \Gamma]^{T}$ such that

$$
\int_{0}^{T} \mathcal{J}(\mathbf{U}) d \tau=\int_{0}^{T}(\boldsymbol{\Theta}, F) d \tau
$$

As an initial step, we observe that

$$
\int_{0}^{T} \mathcal{J}(\mathbf{U}) d \tau=\int_{0}^{T}(\mathbf{U}, G) d \tau-\int_{0}^{T}\left(\boldsymbol{\Theta},(\mathcal{S} \mathbf{U})_{\tau}+(\mathcal{A} \mathbf{U})_{\xi}+(\mathcal{B} \mathbf{U})_{\eta}+\mathcal{C} \mathbf{U}-F\right) d \tau
$$

Next, we add and subtract terms to obtain

$$
\begin{aligned}
\int_{0}^{T} \mathcal{J}(\mathbf{U}) d \tau & =\int_{0}^{T}(\mathbf{U}, G) d \tau-\int_{0}^{T}\left(\boldsymbol{\Theta},(\mathcal{S} \mathbf{U})_{\tau}+(\mathcal{A} \mathbf{U})_{\xi}+(\mathcal{B} \mathbf{U})_{\eta}+\mathcal{C} \mathbf{U}-F\right) d \tau \\
& \pm \int_{0}^{T}\left[\left(\boldsymbol{\Theta}_{\tau}, \mathcal{S} \mathbf{U}\right)+\left(\boldsymbol{\Theta}_{\xi}, \mathcal{A} \mathbf{U}\right)+\left(\boldsymbol{\Theta}_{\eta}, \mathcal{B} \mathbf{U}\right)\right] d \tau
\end{aligned}
$$


One can rearrange (25) as

$$
\begin{aligned}
\int_{0}^{T} \mathcal{J}(\mathbf{U}) d \tau=\int_{0}^{T}(\boldsymbol{\Theta}, F) d \tau & -\int_{0}^{T} \iint_{\Phi}\left(\boldsymbol{\Theta}^{T} \mathcal{S} \mathbf{U}\right)_{\tau} d \xi d \eta d \tau \\
& -\int_{0}^{T} \iint_{\Phi}\left(\left(\boldsymbol{\Theta}^{T} \mathcal{A} \mathbf{U}\right)_{\xi}+\left(\boldsymbol{\Theta}^{T} \mathcal{B} \mathbf{U}\right)_{\eta}\right) d \xi d \eta d \tau \\
& +\int_{0}^{T}\left(\mathcal{S} \boldsymbol{\Theta}_{\tau}+\mathcal{A} \boldsymbol{\Theta}_{\xi}+\mathcal{B} \boldsymbol{\Theta}_{\eta}-\mathcal{C} \boldsymbol{\Theta}+G, \mathbf{U}\right) d \tau
\end{aligned}
$$

Integration by parts together with the use of Green-Gauss theorem leads to

$$
\begin{aligned}
\int_{0}^{T} \mathcal{J}(\mathbf{U}) d \tau & =\int_{0}^{T}(\boldsymbol{\Theta}, F) d \tau-\left.(\boldsymbol{\Theta}, \mathcal{S} \mathbf{U})\right|_{\tau=0} ^{\tau=1}-\int_{0}^{T} \oint_{\delta \Phi} \Theta^{T} D \mathbf{U} d s d \tau \\
& +\int_{0}^{T}\left(\mathcal{S} \Theta_{\tau}+\mathcal{A} \Theta_{\xi}+\mathcal{B} \Theta_{\eta}-\mathcal{C} \Theta+G, \mathbf{U}\right) d \tau
\end{aligned}
$$

By applying the previous eigenvalue decomposition of $E$, and considering the homogeneous version of the initial and boundary conditions for the primal problem given in (21), we obtain

$$
\begin{aligned}
\int_{0}^{T} \mathcal{J}(\mathbf{U}) d \tau & =\int_{0}^{T}(\boldsymbol{\Theta}, F) d \tau-(\boldsymbol{\Theta}, \mathcal{S} \mathbf{U})_{\tau=1}-\int_{0}^{T} \oint_{\delta \Phi}\left(X_{+}^{T} \boldsymbol{\Theta}\right)^{T} \Lambda_{+}\left(X_{+}^{T} \mathbf{U}\right) d s d \tau \\
& +\int_{0}^{T}\left(\mathcal{S} \boldsymbol{\Theta}_{\tau}+\mathcal{A} \boldsymbol{\Theta}_{\xi}+\mathcal{B} \boldsymbol{\Theta}_{\eta}-\mathcal{C} \boldsymbol{\Theta}+G, \mathbf{U}\right) d \tau
\end{aligned}
$$

In order to arrive at (23), the last three terms in (28) must vanish. Therefore, the dual problem is given by

$$
\begin{aligned}
-\mathcal{S} \Theta_{\tau}-\mathcal{A} \Theta_{\xi}-\mathcal{B} \Theta_{\eta}+\mathcal{C} \Theta & =G, & (\xi, \eta) & \in \Phi, \\
X_{+}^{T} \Theta & =0, & (\xi, \eta) & \in \delta \Phi, \\
\Theta(\xi, \eta) & =0, & \tau & =T .
\end{aligned}
$$

We can prove

Proposition 2. The dual problem in (29) is well-posed. 
Proof. By the use of (13) in (29), we can rewrite the dual equation in conservative form as

$$
-(\mathcal{S} \Theta)_{\tau}-(\mathcal{A} \Theta)_{\xi}-(\mathcal{B} \Theta)_{\eta}+\mathcal{C} \Theta=0,(\xi, \eta) \in \Phi, \tau \in[0, T]
$$

where we ignored the forcing function. The energy method applied to (30) and the matrix $D$ decomposed as before, lead to

$$
\begin{array}{r}
-\|\boldsymbol{\Theta}(T, \xi, \eta)\|_{\mathcal{S}(T,)}^{2}+\|\boldsymbol{\Theta}(0, \xi, \eta)\|_{\mathcal{S}(0)}^{2}+2 \int_{0}^{T} \iint_{\Phi} \boldsymbol{\Theta}^{T} \mathcal{C} \boldsymbol{\Theta} d \Phi d \tau= \\
+\int_{0}^{T} \oint_{\delta \Phi}\left(X_{+}^{T} \boldsymbol{\Theta}\right)^{T} \Lambda_{+}\left(X_{+}^{T} \boldsymbol{\Theta}\right) d s d \tau+\int_{0}^{T} \oint_{\delta \Phi}\left(X_{-}^{T} \boldsymbol{\Theta}\right)^{T} \Lambda_{-}\left(X_{-}^{T} \boldsymbol{\Theta}\right) d s d \tau .
\end{array}
$$

With zero initial data and the dual boundary conditions in (29), we obtain

$$
\|\Theta(0, \xi, \eta)\|_{\mathcal{S}(0)}^{2}+2 \int_{0}^{T} \iint_{\Phi} \Theta^{T} \mathcal{C} \Theta d \xi d \eta d \tau-\int_{0}^{T} \oint_{\delta \Phi}\left(X_{-}^{T} \boldsymbol{\Theta}\right)^{T} \Lambda_{-}\left(X_{-}^{T} \boldsymbol{\Theta}\right) d s d \tau=0
$$

By (32), we conclude that the dual problem (29) is well-posed.

For later reference, with only the south boundary term considered, the dual energy estimate becomes

$$
\begin{aligned}
\|\boldsymbol{\Theta}(0, \xi, \eta)\|_{\mathcal{S}(0)}^{2} & +2 \int_{0}^{T} \iint_{\Phi} \boldsymbol{\Theta}^{T} \mathcal{C} \Theta d \xi d \eta d \tau \\
& +\int_{0}^{T} \int\left[\left(X_{\mathcal{B}_{s}}\right)_{+}^{T} \boldsymbol{\Theta}\right]^{T}\left(\Lambda_{\mathcal{B}_{s}}\right)_{+}\left[\left(X_{\mathcal{B}_{s}}\right)_{+}^{T} \boldsymbol{\Theta}\right] d \xi d \tau=0 .
\end{aligned}
$$

\section{The discrete problem}

We discretize $\Phi=[0,1] \times[0,1]$, with a spatial mesh of $N+1$ and $M+1$ grid points in $\xi$ and $\eta$ directions, respectively. In time, we use a mesh of size $K+1$ from $t=0$ to $t=T$. The fully discrete numerical solution is a vector 
of size $9(K+1)(N+1)(M+1)$, arranged as

$$
\tilde{\mathbf{U}}=\left[\begin{array}{c}
\tilde{\mathbf{U}}_{0} \\
\vdots \\
\left.\tilde{\mathbf{U}}_{k}\right] \\
\vdots \\
\tilde{\mathbf{U}}_{K}
\end{array}\right] ;\left[\tilde{\mathbf{U}}_{k}\right]=\left[\begin{array}{c}
\tilde{\mathbf{U}}_{0} \\
\vdots \\
{\left[\tilde{\mathbf{U}}_{n}\right]} \\
\vdots \\
\tilde{\mathbf{U}}_{N}
\end{array}\right]_{k} ;\left[\tilde{\mathbf{U}}_{n}\right]_{k}=\left[\begin{array}{c}
\tilde{\mathbf{U}}_{0} \\
\vdots \\
{\left[\tilde{\mathbf{U}}_{m}\right]} \\
\vdots \\
\tilde{\mathbf{U}}_{M}
\end{array}\right]_{k n} ;\left[\tilde{\mathbf{U}}_{m}\right]_{k n}=\left[\begin{array}{c}
\tilde{U} \\
\tilde{V} \\
\tilde{W}
\end{array}\right]_{k n m}=\tilde{\mathbf{U}}_{k n m},
$$

where $\tilde{\mathbf{U}}_{k n m} \approx \mathbf{U}\left(\tau_{k}, \xi_{n}, \eta_{m}\right)$. A schematic of the spatial mesh at $\tau=\tau_{k}$ and the indexing used is shown in Figure 2.

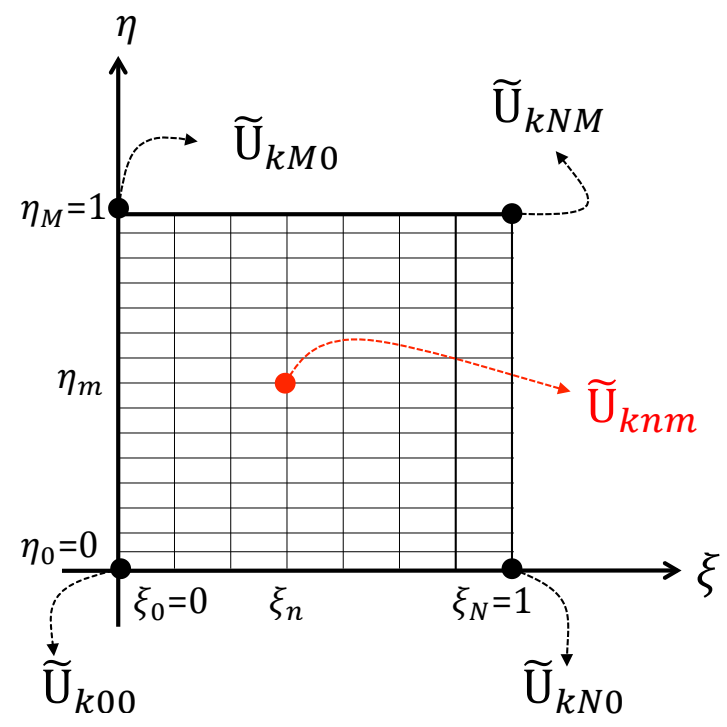

Figure 2: A schematic of the mesh and the indexing used in the arrangement of the numerical solution at $\tau=\tau_{k}$.

The first derivative $\phi_{\xi}$ is approximated by $D_{\xi} \phi$, where $D_{\xi}$ is a so-called SBP operator of the form

$$
D_{\xi}=P_{\xi}^{-1} Q_{\xi},
$$

and $\phi=\left[\phi_{0}, \phi_{1}, \cdots, \phi_{N}\right]^{T}$ is a smooth function injected in each grid point in the $\xi$ direction. The matrix $P_{\xi}$ is symmetric positive definite, and $Q_{\xi}$ is 
almost skew-symmetric satisfying

$$
Q_{\xi}+Q_{\xi}^{T}=B_{\xi}=E_{1}-E_{0}=\operatorname{diag}(-1,0, \ldots, 0,1) .
$$

In $(36), E_{0}=\operatorname{diag}(1,0, \ldots, 0)$ and $E_{1}=\operatorname{diag}(0, \ldots, 0,1)$, both of size $(N+1) \times$ $(N+1)$. The difference operators in $\eta$ and $\tau$ directions, i.e. $D_{\eta}=P_{\eta}^{-1} Q_{\eta}$ of size $(M+1) \times(M+1)$ and $D_{\tau}=P_{\tau}^{-1} Q_{\tau}$ of size $(K+1) \times(K+1)$, are defined in the same way.

A first derivative SBP operator is a $2 s$-order accurate central difference operator which is modified close to the boundaries such that it becomes one-sided. Together with a diagonal norm $P_{\xi, \eta, \tau}$, the boundary closure is $s$-order accurate, making a point-wise stable first order approximation $s+1$ order accurate globally $[1,2,3,14]$. For more details on non-standard SBP operators see $[15,16,17,18]$.

A finite difference operator in multiple space dimensions including the time discretization $[19,20]$, on SBP form, is constructed by extending the one-dimensional SBP operators in a tensor product fashion as

$$
\begin{aligned}
& \mathcal{D}_{\tau}=D_{\tau} \otimes I_{\xi} \otimes I_{\eta} \otimes I, \\
& \mathcal{D}_{\xi}=I_{\tau} \otimes D_{\xi} \otimes I_{\eta} \otimes I, \\
& \mathcal{D}_{\eta}=I_{\tau} \otimes I_{\xi} \otimes D_{\eta} \otimes I .
\end{aligned}
$$

In $(37), \otimes$ represents the Kronecker product [22] which is defined as

$$
A \otimes B=\left[\begin{array}{ccc}
a_{00} B & \ldots & a_{0 m} B \\
\vdots & \ddots & \vdots \\
a_{n 0} B & \ldots & a_{n m} B
\end{array}\right]
$$

for the $(n+1) \times(m+1)$ matrix $A=\left\{a_{i j}\right\}$, and $B$ of arbitrary size. In (37), and in the remainder of this article, all matrices in the first position of the Kronecker products are of size $(K+1) \times(K+1)$, the second position $(N+1) \times(N+1)$, the third position $(M+1) \times(M+1)$ and the fourth position $9 \times 9$. Additionally, $I_{\tau}, I_{\xi}, I_{\eta}$ and I denote the identity matrices with a size consistent with their positions in the Kronecker product.

In (21), the coefficient matrices on the left hand side are variable and symmetric, therefore, prior to constructing the scheme, we apply the splitting technique in [13]. The forcing function on the right hand side of (21) is 
ignored, since it does not affect the stability analysis. The result is

$$
\begin{aligned}
\frac{1}{2}\left((\mathcal{S U})_{\tau}+\mathcal{S} \mathbf{U}_{\tau}+\mathcal{S}_{\tau} \mathbf{U}\right) & +\frac{1}{2}\left((\mathcal{A U})_{\xi}+\mathcal{A} \mathbf{U}_{\xi}+\mathcal{A}_{\xi} \mathbf{U}\right) \\
& +\frac{1}{2}\left((\mathcal{B} \mathbf{U})_{\eta}+\mathcal{B} \mathbf{U}_{\eta}+\mathcal{B}_{\eta} \mathbf{U}\right)+\mathcal{C} \mathbf{U}=0
\end{aligned}
$$

The fully discrete version of (39) on SBP-SAT form, including weakly imposed initial and boundary conditions, is

$$
\begin{aligned}
\frac{1}{2}\left[\mathcal{D}_{\tau} \tilde{\mathcal{S}}+\tilde{\mathcal{S}} \mathcal{D}_{\tau}+\tilde{\mathcal{S}}_{\tau}\right] \tilde{\mathbf{U}} & +\frac{1}{2}\left[\mathcal{D}_{\xi} \tilde{\mathcal{A}}+\tilde{\mathcal{A}} \mathcal{D}_{\xi}+\tilde{\mathcal{A}}_{\xi}\right] \tilde{\mathbf{U}}+\frac{1}{2}\left[\mathcal{D}_{\eta} \tilde{\mathcal{B}}+\tilde{\mathcal{B}} \mathcal{D}_{\eta}+\tilde{\mathcal{B}}_{\eta}\right] \tilde{\mathbf{U}}+\tilde{\mathcal{C}} \tilde{\mathbf{U}} \\
& =\mathcal{P}_{i}^{-1} \Sigma_{i}(\tilde{\mathbf{U}}-\mathbf{f})+\mathcal{P}_{s}^{-1} \Sigma_{s}\left[\left(\tilde{X}_{\mathcal{B}_{s}}\right)_{+}^{T} \tilde{\mathbf{U}}-\left(\tilde{X}_{\mathcal{B}_{s}}\right)_{+}^{T} \mathbf{U}_{\infty}\right]
\end{aligned}
$$

In $(40), \mathcal{P}_{i}^{-1}=P_{\tau}^{-1} E_{0} \otimes I_{\xi} \otimes I_{\eta} \otimes I$ and $\mathcal{P}_{s}^{-1}=I_{\tau} \otimes I_{\xi} \otimes P_{\eta}^{-1} E_{0} \otimes I$ where the subscripts $i$ and $s$ indicate initial and south, respectively. We have only considered the south boundary procedure in (40), since the treatment of other boundaries is similar. Additionally in (40), $\mathbf{U}_{\infty}$ and $\mathbf{f}$, are vectors containing boundary and initial data at the relevant positions.

In $(40), \tilde{\mathcal{S}}, \tilde{\mathcal{S}}_{\tau}, \tilde{\mathcal{A}}, \tilde{\mathcal{A}}_{\xi}, \tilde{\mathcal{B}}, \tilde{\mathcal{B}}_{\eta}, \tilde{\mathcal{C}}$ and $\tilde{X}_{\mathcal{B}_{s}}$ are block diagonal matrices that approximate $\mathcal{S}, \mathcal{S}_{\tau}, \mathcal{A}, \mathcal{A}_{\xi}, \mathcal{B}, \mathcal{B}_{\eta}, \mathcal{C}$ and $X_{\mathcal{B}_{s}}$ point wise in the following way

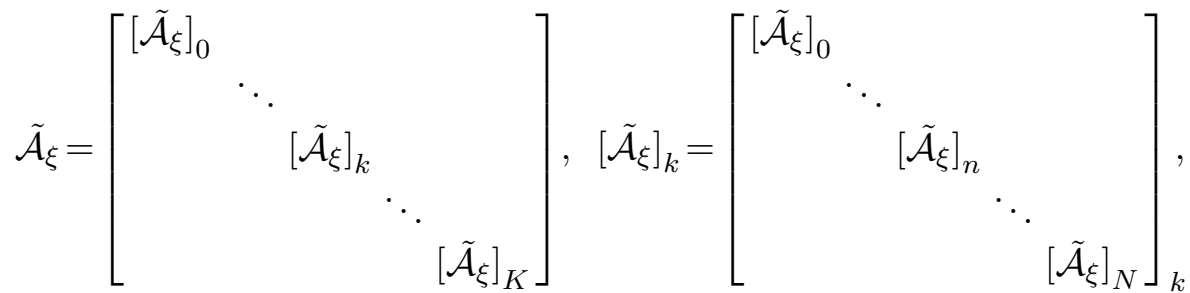

$$
\begin{aligned}
& {\left[\tilde{\mathcal{A}}_{\xi}\right]_{k n}=\left[\begin{array}{ccccc}
{\left[\tilde{\mathcal{A}}_{\xi}\right]_{0}} & & & & \\
& \ddots & & & \\
& & {\left[\tilde{\mathcal{A}}_{\xi}\right]_{m}} & & \\
& & & \ddots & \\
& & & {\left[\tilde{\mathcal{A}}_{\xi}\right]_{M}}
\end{array}\right]_{k n}}
\end{aligned}
$$

The matrices $\tilde{\mathcal{S}}, \tilde{\mathcal{S}}_{\tau}, \tilde{\mathcal{A}}, \tilde{\mathcal{A}}_{\xi}, \tilde{\mathcal{B}}, \tilde{\mathcal{B}}_{\eta}$ and $\tilde{\mathcal{C}}$ include numerical approximations of the metric terms. 
We use

$$
\begin{aligned}
\tilde{\mathcal{S}} & =\bar{J}\left(I_{\tau} \otimes I_{\xi} \otimes I_{\eta} \otimes \mathbf{S}\right) \\
\tilde{\mathcal{A}} & =\overline{J \xi_{t}}+\overline{J \xi_{x}}\left(I_{\tau} \otimes I_{\xi} \otimes I_{\eta} \otimes \overline{\mathbf{A}}\right)+\overline{J \xi_{y}}\left(I_{\tau} \otimes I_{\xi} \otimes I_{\eta} \otimes \overline{\mathbf{B}}\right) \\
\tilde{\mathcal{B}} & =\overline{J \eta_{t}}+\overline{J \eta_{x}}\left(I_{\tau} \otimes I_{\xi} \otimes I_{\eta} \otimes \overline{\mathbf{A}}\right)+\overline{J \eta_{y}}\left(I_{\tau} \otimes I_{\xi} \otimes I_{\eta} \otimes \overline{\mathbf{B}}\right)
\end{aligned}
$$

where $\bar{J}, \overline{J \xi_{t}}, \overline{J \xi_{x}}, \overline{J \xi_{y}}, \overline{J \eta_{t}}, \overline{J \eta_{x}}$ and $\overline{J \eta_{y}}$ are diagonal matrices, containing point wise approximations of $J, J \xi_{t}, J \xi_{x}, J \xi_{y}, J \eta_{t}, J \eta_{x}$ and $J \eta_{y}$, respectively. The numerical metric terms are defined as

$$
\begin{aligned}
& \bar{J}=\operatorname{diag}\left[\mathcal{D}_{\eta} M^{(1)}-\mathcal{D}_{\xi} M^{(2)}\right] \\
& \overline{J \xi_{t}}=\operatorname{diag}\left[\mathcal{D}_{\tau} M^{(2)}-\mathcal{D}_{\xi} M^{(3)}\right], \quad \overline{J \xi_{x}}=\operatorname{diag}\left[\mathcal{D}_{\eta} \mathbf{y}\right], \quad \overline{J \xi_{y}}=-\operatorname{diag}\left[\mathcal{D}_{\eta} \mathbf{x}\right], \\
& \overline{J \eta_{t}}=\operatorname{diag}\left[\mathcal{D}_{\xi} M^{(3)}-\mathcal{D}_{\tau} M^{(1)}\right], \quad \overline{J \eta_{x}}=-\operatorname{diag}\left[\mathcal{D}_{\xi} \mathbf{y}\right], \quad \overline{J \eta_{y}}=-\operatorname{diag}\left[\mathcal{D}_{\xi} \mathbf{x}\right] .
\end{aligned}
$$

where $\mathbf{x}, \mathbf{y}$ are vectors containing the $\mathbf{x}$ and $\mathrm{y}$ coordinates of the Cartesian mesh arranged similar to the numerical solution in (34). Moreover,

$$
M^{(1)}=\operatorname{diag}(\mathbf{y}) \mathcal{D}_{\xi} \mathbf{x}, \quad M^{(2)}=\operatorname{diag}(\mathbf{y}) \mathcal{D}_{\eta} \mathbf{x}, \quad M^{(3)}=\operatorname{diag}(\mathbf{y}) \mathcal{D}_{\tau} \mathbf{x}
$$

and

$$
\begin{aligned}
& \tilde{\mathcal{S}}_{\tau}=\left(\mathcal{D}_{\tau} \bar{J}\right)\left(I_{\tau} \otimes I_{\xi} \otimes I_{\eta} \otimes \mathbf{S}\right) \\
& \tilde{\mathcal{A}}_{\xi}=\left(\mathcal{D}_{\xi} \overline{J \xi_{t}}\right)+\left(\mathcal{D}_{\xi} \overline{J \xi_{x}}\right)\left(I_{\tau} \otimes I_{\xi} \otimes I_{\eta} \otimes \overline{\mathbf{A}}\right)+\left(\mathcal{D}_{\xi} \overline{J \xi_{y}}\right)\left(I_{\tau} \otimes I_{\xi} \otimes I_{\eta} \otimes \overline{\mathbf{B}}\right) \\
& \tilde{\mathcal{B}}_{\eta}=\left(\mathcal{D}_{\eta} \overline{J \eta_{t}}\right)+\left(\mathcal{D}_{\eta} \overline{J \eta_{x}}\right)\left(I_{\tau} \otimes I_{\xi} \otimes I_{\eta} \otimes \mathbf{A}\right)+\left(\mathcal{D}_{\eta} \overline{J \eta_{y}}\right)\left(I_{\tau} \otimes I_{\xi} \otimes I_{\eta} \otimes \overline{\mathbf{B}}\right)
\end{aligned}
$$

Furthermore, in (40), $\Sigma_{s}$ is the penalty matrix corresponding to the weak imposition of the south boundary condition, arranged as

$$
\begin{aligned}
& \Sigma_{s}=\left[\begin{array}{ccccc}
{\left[\Sigma_{s}\right]_{0}} & & & & \\
& \ddots & & & \\
& & {\left[\Sigma_{s}\right]_{k}} & & \\
& & & \ddots & \\
& & & & {\left[\Sigma_{s}\right]_{K}}
\end{array}\right]
\end{aligned}
$$

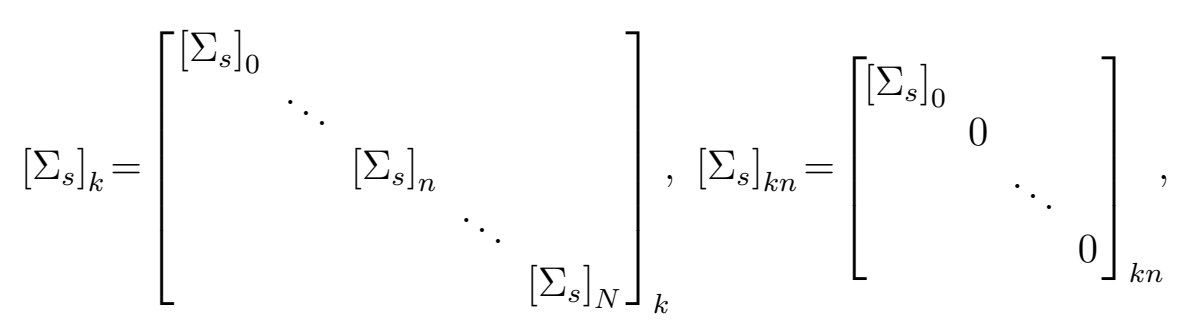


where $\left[\Sigma_{s}\right]_{k n 0}$ operates on the south boundary i.e. $\left(\xi_{n}, \eta_{0}\right)$ for $n \in\{0, \ldots, N\}$, see Figure 2.

Finally, in (40), $\Sigma_{i}$ is the penalty operator for the weak imposition of the initial condition arranged as

$$
\begin{aligned}
& \Sigma_{i}=\left[\begin{array}{cccc}
{\left[\Sigma_{i}\right]_{0}} & & & \\
& 0 & & \\
& & \ddots & \\
& & & 0
\end{array}\right] ;
\end{aligned}
$$

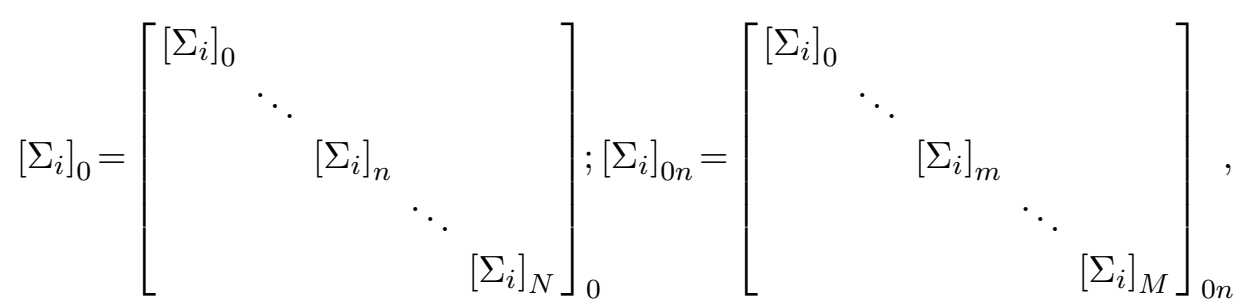

where $\left[\Sigma_{i}\right]_{0 n m}$ operates on the mesh point $\left(\xi_{n}, \eta_{m}\right)$, see Figure 2 , at $\tau=0$.

Lemma 1. By using the definitions given in (43), (44) and (45), the Numerical Geometric Conservation Law (NGCL) holds and results in

$$
\tilde{\mathcal{S}}_{\tau}+\tilde{\mathcal{A}}_{\xi}+\tilde{\mathcal{B}}_{\eta}=0
$$

See [4] for the proof.

\subsection{Stability of the primal problem}

The discrete energy method applied to (40) yields

$$
\begin{aligned}
& \tilde{\mathbf{U}}^{T}\left[B_{\tau} \otimes P_{\xi} \otimes P_{\eta} \otimes I\right] \tilde{\mathcal{S}} \tilde{\mathbf{U}}+\tilde{\mathbf{U}}^{T}\left[P_{\tau} \otimes B_{\xi} \otimes P_{\eta} \otimes I\right] \tilde{\mathcal{A}} \tilde{\mathbf{U}}+ \\
& \tilde{\mathbf{U}}^{T}\left[P_{\tau} \otimes P_{\xi} \otimes B_{\eta} \otimes I\right] \tilde{\mathcal{B}} \tilde{\mathbf{U}}+\tilde{\mathbf{U}}^{T} \mathcal{P}\left(\tilde{\mathcal{S}}_{\tau}+\tilde{\mathcal{A}}_{\xi}+\tilde{\mathcal{B}}_{\eta}\right) \tilde{\mathbf{U}}+2 \tilde{\mathbf{U}}{ }^{T} \mathcal{P} \tilde{\mathcal{C}} \tilde{\mathbf{U}}= \\
& \tilde{\mathbf{U}}^{T}\left(E_{0} \otimes P_{\xi} \otimes P_{\eta} \otimes I\right) \Sigma_{i}(\tilde{\mathbf{U}}-\mathbf{f})+(\tilde{\mathbf{U}}-\mathbf{f})^{T} \Sigma_{i}\left(E_{0} \otimes P_{\xi} \otimes P_{\eta} \otimes I\right) \tilde{\mathbf{U}}+ \\
& \tilde{\mathbf{U}}^{T}\left(P_{\tau} \otimes P_{\xi} \otimes E_{0} \otimes I\right) \Sigma_{s}\left[\left(\tilde{X}_{\mathcal{B}_{s}}\right)_{+}^{T} \tilde{\mathbf{U}}-\left(\tilde{X}_{\mathcal{B}_{s}}\right)_{+}^{T} \mathbf{U}_{\infty}\right]+ \\
& {\left[\left(\tilde{X}_{\mathcal{B}_{s}}\right)_{+}^{T} \tilde{\mathbf{U}}-\left(\tilde{X}_{\mathcal{B}_{s}}\right)_{+}^{T} \mathbf{U}_{\infty}\right]^{T} \Sigma_{s}^{T}\left(P_{\tau} \otimes P_{\xi} \otimes E_{0} \otimes I\right) \tilde{\mathbf{U}}}
\end{aligned}
$$


where $\left.\mathcal{P}=P_{\tau} \otimes P_{\xi} \otimes P_{\eta} \otimes I\right)$. The use of the NGCL (48) and only considering the south boundary terms lead to

$$
\begin{aligned}
& \tilde{\mathbf{U}}^{T}\left(E_{1} \otimes P_{\xi} \otimes P_{\eta} \otimes I\right) \tilde{\mathcal{S}} \tilde{\mathbf{U}}+2 \tilde{\mathbf{U}}^{T} \mathcal{P} \tilde{\mathcal{C}} \tilde{\mathbf{U}}= \\
& \tilde{\mathbf{U}}^{T}\left(E_{0} \otimes P_{\xi} \otimes P_{\eta} \otimes I\right)\left(\tilde{\mathcal{S}}+2 \Sigma_{i}\right) \tilde{\mathbf{U}}-\tilde{\mathbf{U}}^{T}\left(E_{0} \otimes P_{\xi} \otimes P_{\eta} \otimes I\right) \Sigma_{i} \mathbf{f}- \\
& \mathbf{f}^{T} \Sigma_{i}\left(E_{0} \otimes P_{\xi} \otimes P_{\eta} \otimes I\right) \tilde{\mathbf{U}}+\tilde{\mathbf{U}}^{T}\left(P_{\tau} \otimes P_{\xi} \otimes E_{0} \otimes I\right) \tilde{\mathcal{B}_{s}} \tilde{\mathbf{U}}+ \\
& \tilde{\mathbf{U}}^{T}\left(P_{\tau} \otimes P_{\xi} \otimes E_{0} \otimes I\right) \Sigma_{s}\left[\left(\tilde{X}_{\mathcal{B}_{s}}\right)_{+}^{T} \tilde{\mathbf{U}}-\left(\tilde{X}_{\mathcal{B}_{s}}\right)_{+}^{T} \mathbf{U}_{\infty}\right]+ \\
& {\left[\left(\tilde{X}_{\mathcal{B}_{s}}\right)_{+}^{T} \tilde{\mathbf{U}}-\left(\tilde{X}_{\mathcal{B}_{s}}\right)_{+}^{T} \mathbf{U}_{\infty}\right]^{T} \Sigma_{s}^{T}\left(P_{\tau} \otimes P_{\xi} \otimes E_{0} \otimes I\right) \tilde{\mathbf{U}}}
\end{aligned}
$$

The decomposition $\tilde{\mathcal{B}}_{s}=\tilde{X}_{\mathcal{B}_{s}} \tilde{\Lambda}_{\mathcal{B}_{s}} \tilde{X}_{\mathcal{B}_{s}}^{T}$ and $\Sigma_{s}=\left(\tilde{X}_{\mathcal{B}_{s}}\right)_{+} \hat{\Sigma}_{s}$ inserted in (50) gives

$$
\begin{aligned}
& \|\left.\tilde{\mathbf{U}}\right|_{\left(E_{1} \otimes P_{\xi} \otimes P_{\eta} \otimes I\right) \tilde{\mathcal{S}}} ^{2}+2 \tilde{\mathbf{U}}^{T} \mathcal{P} \tilde{\mathcal{C}} \tilde{\mathbf{U}}=\tilde{\mathbf{U}}^{T}\left(E_{0} \otimes P_{\xi} \otimes P_{\eta} \otimes I\right)\left(\tilde{\mathcal{S}}+2 \Sigma_{i}\right) \tilde{\mathbf{U}}- \\
& \tilde{\mathbf{U}}^{T}\left(E_{0} \otimes P_{\xi} \otimes P_{\eta} \otimes I\right) \Sigma_{i} \mathbf{f}-\mathbf{f}^{T} \Sigma_{i}\left(E_{0} \otimes P_{\xi} \otimes P_{\eta} \otimes I\right) \tilde{\mathbf{U}}+ \\
& {\left[\left(\tilde{X}_{\mathcal{B}_{s}}\right)_{-}^{T} \tilde{\mathbf{U}}\right]^{T}\left(P_{\tau} \otimes P_{\xi} \otimes E_{0} \otimes I\right)\left(\tilde{\Lambda}_{\mathcal{B}_{s}}\right)_{-}\left[\left(\tilde{X}_{\mathcal{B}_{s}}\right)_{-}^{T} \tilde{\mathbf{U}}\right]+} \\
& {\left[\left(\tilde{X}_{\mathcal{B}_{s}}\right)_{+}^{T} \tilde{\mathbf{U}}\right]^{T}\left(P_{\tau} \otimes P_{\xi} \otimes E_{0} \otimes I\right)\left[\left(\tilde{\Lambda}_{\mathcal{B}_{s}}\right)_{+}+2 \hat{\Sigma}_{s}\right]\left[\left(\tilde{X}_{\mathcal{B}_{s}}\right)_{+}^{T} \tilde{\mathbf{U}}\right]-} \\
& {\left[\left(\tilde{X}_{\mathcal{B}_{s}}\right)_{+}^{T} \tilde{\mathbf{U}}\right]^{T}\left(P_{\tau} \otimes P_{\xi} \otimes E_{0} \otimes I\right) \hat{\Sigma}_{s}\left[\left(\tilde{X}_{\mathcal{B}_{s}}\right)_{+}^{T} \mathbf{U}_{\infty}\right]-} \\
& {\left[\left(\tilde{X}_{\mathcal{B}_{s}}\right)_{+}^{T} \tilde{\mathbf{U}}_{\infty}\right]^{T} \hat{\Sigma}_{s}\left(P_{\tau} \otimes P_{\xi} \otimes E_{0} \otimes I\right)\left[\left(\tilde{X}_{\mathcal{B}_{s}}\right)_{+}^{T} \tilde{\mathbf{U}}\right]}
\end{aligned}
$$

where

$$
\|\tilde{\mathbf{U}}\|_{\left(E_{1} \otimes P_{\xi} \otimes P_{\eta} \otimes I\right) \tilde{\mathcal{S}}}^{2}=\tilde{\mathbf{U}}^{T}\left(E_{1} \otimes P_{\xi} \otimes P_{\eta} \otimes I\right) \tilde{\mathcal{S}} \tilde{\mathbf{U}}^{T} \approx \iint_{\Phi} J\left(u^{2}+v^{2}\right) d \xi d \eta .
$$

To find stability requirements, it is convenient to consider zero data in 
(51). Zero energy growth is obtained under the conditions

$$
\begin{aligned}
& \Sigma_{i} \leq-\tilde{\mathcal{S}} / 2, \\
& \left(\hat{\Sigma}_{s}\right)_{j j} \leq-\left(\tilde{\Lambda}_{\mathcal{B}_{s}}\right)_{j j} / 2 \text { if }\left(\tilde{\Lambda}_{\mathcal{B}_{s}}\right)_{j j}>0 \text {, } \\
& \left(\hat{\Sigma}_{s}\right)_{j j}=0 \quad \text { if }\left(\tilde{\Lambda}_{\mathcal{B}_{s}}\right)_{j j} \leq 0 \text {, }
\end{aligned}
$$

for $j \in\{0, \ldots, 9(K+1)(N+1)(M+1)\}$.

\subsection{Stability of the dual problem}

Similar to the primal case, the splitting technique is applied to the dual problem in (29), prior to constructing the scheme. The result is

$$
\begin{aligned}
-\frac{1}{2}\left[(\mathcal{S} \Theta)_{\tau}+\mathcal{S} \Theta_{\tau}+\mathcal{S}_{\tau} \Theta\right] & -\frac{1}{2}\left[(\mathcal{A} \Theta)_{\xi}+\mathcal{A} \Theta_{\xi}+\mathcal{A}_{\xi} \Theta\right] \\
& -\frac{1}{2}\left[(\mathcal{B} \Theta)_{\eta}+\mathcal{B} \Theta_{\eta}+\mathcal{B}_{\eta} \Theta\right]+\mathcal{C} \Theta=0,
\end{aligned}
$$

where we ignored the forcing function. Next, we discretize (54), as in the primal case, and weakly impose the homogeneous final time and boundary conditions given in (29). Again, we only consider the south boundary procedure. The discrete dual problem becomes

$$
\begin{aligned}
-\frac{1}{2}\left[D_{\tau} \tilde{\mathcal{S}}+\tilde{\mathcal{S}} D_{\tau}+\tilde{\mathcal{S}}_{\tau}\right] \tilde{\boldsymbol{\Theta}}-\frac{1}{2}\left[D_{\xi} \tilde{\mathcal{A}}+\tilde{\mathcal{A}} D_{\xi}+\tilde{\mathcal{A}}_{\xi}\right] \tilde{\boldsymbol{\Theta}}-\frac{1}{2}\left[D_{\eta} \tilde{\mathcal{B}}+\tilde{\mathcal{B}} D_{\eta}+\tilde{\mathcal{B}}_{\eta}\right] \tilde{\boldsymbol{\Theta}}+\tilde{\mathcal{C}} \tilde{\boldsymbol{\Theta}} \\
=\mathcal{P}_{f}^{-1} \Sigma_{f} \tilde{\boldsymbol{\Theta}}+\mathcal{P}_{s}^{-1} \Sigma_{s}^{d}\left[\left(\tilde{X}_{\mathcal{B}_{s}}\right)_{+}^{T} \tilde{\boldsymbol{\Theta}}\right] .
\end{aligned}
$$

In (55), $\mathcal{P}_{f}^{-1}=P_{\tau}^{-1} E_{1} \otimes I_{\xi} \otimes I_{\eta} \otimes I, \Sigma_{f}$ and $\Sigma_{s}^{d}$ are the penalty operators corresponding to the weak final time and south boundary procedure of the dual problem, respectively. We consider $\Sigma_{s}^{d}=\left(\tilde{X}_{\mathcal{B}_{s}}\right)_{-} \hat{\Sigma}_{s}^{d}$, where $\hat{\Sigma}_{s}^{d}$ is diagonal.

We use the discrete energy method (multiplying (55) with $\tilde{\Theta}^{T} \mathcal{P}$ ) to study the stability of the dual problem. This procedure is identical to the stability analysis for the primal problem, and therefore not repeated here. Stability of the dual problem require

$$
\begin{aligned}
\Sigma_{f} & \leq-\tilde{\mathcal{S}} / 2, & & \\
\left(\hat{\Sigma}_{s}^{d}\right)_{j j} & \leq\left(\tilde{\Lambda}_{\mathcal{B}_{s}}\right)_{j j} / 2 & \text { if } & \left(\tilde{\Lambda}_{\mathcal{B}_{s}}\right)_{j j}<0, \\
\left(\hat{\Sigma}_{s}^{d}\right)_{j j} & =0 & \text { if } \quad & \left(\tilde{\Lambda}_{\mathcal{B}_{s}}\right)_{j j} \geq 0,
\end{aligned}
$$

where $j \in\{0, \ldots, 9(K+1)(N+1)(M+1)\}$. 


\subsection{The dual consistent approximation}

So far, we have determined conditions for the penalty operators $\left(\Sigma_{i}, \Sigma_{f}, \Sigma_{s}\right.$ and $\Sigma_{s}^{d}$ ), given in (53) and (56), that lead to stable primal (40) and dual (55) approximations. Now, we aim for the specific choices of the penalty operators, that make the approximations dual consistent. The procedure is as follows:

1. We consider zero initial and boundary data in (40) and add a non-zero forcing function, $\mathbf{F}$, to the right hand side. The result can be rewritten in form of

$$
\mathcal{L} \tilde{\mathbf{U}}=\mathcal{P} \mathbf{F},
$$

where $\mathcal{L}$ contains the SBP-SAT contributions.

2. We consider zero initial and boundary data in (55) and add a non-zero forcing function, $\mathbf{G}$, to the right hand side. The result can be rewritten as

$$
\mathcal{L}^{d} \tilde{\boldsymbol{\Theta}}=P \mathbf{G},
$$

where $\mathcal{L}^{d}$ contains the SBP-SAT contributions.

3. A dual consistent approximation is obtained if

$$
\mathcal{L}=\left(\mathcal{L}^{d}\right)^{T}
$$

by which we can find the specific values of the penalty operators.

The operator $\mathcal{L}$ in $(57)$ is

$$
\begin{aligned}
\mathcal{L}= & \frac{1}{2}\left[\left(Q_{\tau} \otimes P_{\xi} \otimes P_{\eta} \otimes I\right) \tilde{\mathcal{S}}+\tilde{\mathcal{S}}\left(Q_{\tau} \otimes P_{\xi} \otimes P_{\eta} \otimes I\right)+\tilde{\mathcal{S}}_{\tau}\right]+ \\
& \frac{1}{2}\left[\left(P_{\tau} \otimes Q_{\xi} \otimes P_{\eta} \otimes I\right) \tilde{\mathcal{A}}+\tilde{\mathcal{A}}\left(P_{\tau} \otimes Q_{\xi} \otimes P_{\eta} \otimes I\right)+\tilde{\mathcal{A}}_{\xi}\right]+ \\
& \frac{1}{2}\left[\left(P_{\tau} \otimes P_{\xi} \otimes Q_{\eta} \otimes I\right) \tilde{\mathcal{B}}+\tilde{\mathcal{B}}\left(P_{\tau} \otimes P_{\xi} \otimes Q_{\eta} \otimes I\right)+\tilde{\mathcal{B}}_{\eta}\right]+ \\
& \mathcal{P} \tilde{\mathcal{C}}-\left(E_{0} \otimes P_{\xi} \otimes P_{\eta} \otimes I\right) \Sigma_{i}-\left(P_{\tau} \otimes P_{\xi} \otimes E_{0} \otimes I\right) \Sigma_{s}\left(\tilde{X}_{\mathcal{B}_{s}}\right)_{+}^{T},
\end{aligned}
$$


and the operator $\mathcal{L}^{d}$ in $(58)$ is

$$
\begin{aligned}
\mathcal{L}^{d}= & -\frac{1}{2}\left[\left(Q_{\tau} \otimes P_{\xi} \otimes P_{\eta} \otimes I\right) \tilde{\mathcal{S}}+\tilde{\mathcal{S}}\left(Q_{\tau} \otimes P_{\xi} \otimes P_{\eta} \otimes I\right)+\tilde{\mathcal{S}}_{\tau}\right] \\
& -\frac{1}{2}\left[\left(P_{\tau} \otimes Q_{\xi} \otimes P_{\eta} \otimes I\right) \tilde{\mathcal{A}}+\tilde{\mathcal{A}}\left(P_{\tau} \otimes Q_{\xi} \otimes P_{\eta} \otimes I\right)+\tilde{\mathcal{A}}_{\xi}\right] \\
& -\frac{1}{2}\left[\left(P_{\tau} \otimes P_{\xi} \otimes Q_{\eta} \otimes I\right) \tilde{\mathcal{B}}+\tilde{\mathcal{B}}\left(P_{\tau} \otimes P_{\xi} \otimes Q_{\eta} \otimes I\right)+\tilde{\mathcal{B}}_{\eta}\right] \\
& +\mathcal{P} \tilde{\mathcal{C}}-\left(E_{1} \otimes P_{\xi} \otimes P_{\eta} \otimes I\right) \Sigma_{f}-\left(P_{\tau} \otimes P_{\xi} \otimes E_{0} \otimes I\right) \Sigma_{s}^{d}\left(\tilde{X}_{\mathcal{B}_{s}}\right)_{-}^{T} .
\end{aligned}
$$

Subtracting (60) from the transpose of (61), using the NGCL given in Lemma (1) and the SBP property (36) give

$$
\begin{aligned}
\left(\mathcal{L}^{d}\right)^{T}-\mathcal{L}= & -\frac{1}{2}\left[\left(B_{\tau} \otimes P_{\xi} \otimes P_{\eta} \otimes I\right) \tilde{\mathcal{S}}+\tilde{\mathcal{S}}\left(B_{\tau} \otimes P_{\xi} \otimes P_{\eta} \otimes I\right)\right] \\
& -\frac{1}{2}\left[\left(P_{\tau} \otimes B_{\xi} \otimes P_{\eta} \otimes I\right) \tilde{\mathcal{A}}+\tilde{\mathcal{A}}\left(P_{\tau} \otimes B_{\xi} \otimes P_{\eta} \otimes I\right)\right] \\
& -\frac{1}{2}\left[\left(P_{\tau} \otimes P_{\xi} \otimes B_{\eta} \otimes I\right) \tilde{\mathcal{B}}+\tilde{\mathcal{B}}\left(P_{\tau} \otimes P_{\xi} \otimes B_{\eta} \otimes I\right)\right] \\
& -\Sigma_{f}\left(E_{1} \otimes P_{\xi} \otimes P_{\eta} \otimes I\right)-\left(\tilde{X}_{\mathcal{B}_{s}}\right)_{-}\left(\Sigma_{s}^{d}\right)^{T}\left(P_{\tau} \otimes P_{\xi} \otimes E_{0} \otimes I\right) \\
& +\left(E_{0} \otimes P_{\xi} \otimes P_{\eta} \otimes I\right) \Sigma_{i}+\left(P_{\tau} \otimes P_{\xi} \otimes E_{0} \otimes I\right) \Sigma_{s}\left(\tilde{X}_{\mathcal{B}_{s}}\right)_{+}^{T} \cdot
\end{aligned}
$$

By considering only the terms corresponding to the initial and final time and the south boundary in (62), we obtain

$$
\begin{aligned}
\left(\mathcal{L}^{d}\right)^{T}-\mathcal{L}=- & {\left[\left(E_{1}-E_{0}\right) \otimes P_{\xi} \otimes P_{\eta} \otimes I\right] \tilde{\mathcal{S}}+\left(P_{\tau} \otimes P_{\xi} \otimes E_{0} \otimes I\right) \tilde{\mathcal{B}} } \\
& -\Sigma_{f}\left(E_{1} \otimes P_{\xi} \otimes P_{\eta} \otimes I\right)-\left(\tilde{X}_{\mathcal{B}_{s}}\right)_{-}\left(\Sigma_{s}^{d}\right)^{T}\left(P_{\tau} \otimes P_{\xi} \otimes E_{0} \otimes I\right) \\
& +\left(E_{0} \otimes P_{\xi} \otimes P_{\eta} \otimes I\right) \Sigma_{i}+\left(P_{\tau} \otimes P_{\xi} \otimes E_{0} \otimes I\right) \Sigma_{s}\left(\tilde{X}_{\mathcal{B}_{s}}\right)_{+}^{T}+R .
\end{aligned}
$$


In (63), $R$ includes the contribution of other boundaries than the south. To secure (59) we need

$$
\begin{aligned}
& \Sigma_{i}=-\tilde{\mathcal{S}} \\
& \hat{\Sigma}_{s}=-\frac{\tilde{\Lambda}_{\tilde{\mathcal{B}}_{s}}+\left|\tilde{\Lambda}_{\tilde{\mathcal{B}}_{s}}\right|}{2}=-\left(\tilde{\Lambda}_{\mathcal{B}_{s}}\right)_{+} \\
& \Sigma_{f}=-\tilde{\mathcal{S}} \\
& \hat{\Sigma}_{s}^{d}=\frac{\tilde{\Lambda}_{\mathcal{B}_{s}}-\left|\tilde{\Lambda}_{\tilde{\mathcal{B}}_{s}}\right|}{2}=\left(\tilde{\Lambda}_{\mathcal{B}_{s}}\right)_{-} .
\end{aligned}
$$

Note that the results in (64) satisfy the stability conditions in (53) and (56).

Finally, we substitute the primal penalty operators in (64) into (51) and consider non-zero initial and boundary data. The result is

$$
\begin{aligned}
\|\tilde{\mathbf{U}}\|_{\left(E_{1} \otimes P_{\xi} \otimes P_{\eta} \otimes I\right) \tilde{\mathcal{S}}}^{2} & -\left[\left(\tilde{X}_{\mathcal{B}_{s}}\right)_{-}^{T} \tilde{\mathbf{U}}\right]^{T}\left(P_{\tau} \otimes P_{\xi} \otimes E_{0} \otimes I\right)\left(\tilde{\Lambda}_{\mathcal{B}_{s}}\right)_{-}\left[\left(\tilde{X}_{\mathcal{B}_{s}}\right)_{-}^{T} \tilde{\mathbf{U}}\right]+2 \tilde{\mathbf{U}}^{T} \mathcal{P} \tilde{\mathcal{C}} \tilde{\mathbf{U}} \\
= & \|\mathbf{f}\|_{\left(E_{0} \otimes P_{\xi} \otimes P_{\eta} \otimes I\right) \tilde{\mathcal{S}}}^{2}-\|\tilde{\mathbf{U}}-\mathbf{f}\|_{\left(E_{0} \otimes P_{\xi} \otimes P_{\eta} \otimes I\right) \tilde{\mathcal{S}}}^{2} \\
& +\left[\left(\tilde{X}_{\mathcal{B}_{s}}\right)_{+}^{T} \mathbf{U}_{\infty}\right]^{T}\left(P_{\tau} \otimes P_{\xi} \otimes E_{0} \otimes I\right)\left(\tilde{\Lambda}_{\mathcal{B}_{s}}\right)_{+}\left[\left(\tilde{X}_{\mathcal{B}_{s}}\right)_{+}^{T} \mathbf{U}_{\infty}\right] \\
& -\left[\left(\tilde{X}_{\mathcal{B}_{s}}\right)_{+}^{T}\left(\tilde{\mathbf{U}}-\mathbf{U}_{\infty}\right)\right]^{T}\left(P_{\tau} \otimes P_{\xi} \otimes E_{0} \otimes I\right)\left(\tilde{\Lambda}_{\mathcal{B}_{s}}\right)_{+}\left[\left(\tilde{X}_{\mathcal{B}_{s}}\right)_{+}^{T}\left(\tilde{\mathbf{U}}-\mathbf{U}_{\infty}\right)\right] .
\end{aligned}
$$

Note that the estimate in (65) mimics the continuous counterpart in (20).

Similarly, substituting the dual penalty operators in (64) to (55) leads to $\|\tilde{\boldsymbol{\Theta}}\|_{\left(E_{0} \otimes P_{\xi} \otimes P_{\eta} \otimes I\right) \tilde{\mathcal{S}}}^{2}\left[\left(\tilde{X}_{\mathcal{B}_{s}}\right)_{+}^{T} \tilde{\mathbf{\Theta}}\right]^{T}\left(P_{\tau} \otimes P_{\xi} \otimes E_{0} \otimes I\right)\left(\tilde{\Lambda}_{\mathcal{B}_{s}}\right)_{+}\left[\left(\tilde{X}_{\mathcal{B}_{s}}\right)_{+}^{T} \tilde{\mathbf{\Theta}}\right]^{T}+2 \tilde{\mathbf{\Theta}}^{T} \mathcal{P} \tilde{\mathcal{C}} \tilde{\boldsymbol{\Theta}}=0$.

The dual estimate in (66) mimics the continuous counterpart given in (33).

\section{Numerical experiments}

We consider (5) with $(\bar{u}, \bar{v})=(1,1)$ and $\mu=0.1$ posed on a deforming domain where the boundaries coincide with segments of constant polar coordinates. The coordinate transformation is shown schematically in Figure 3. 


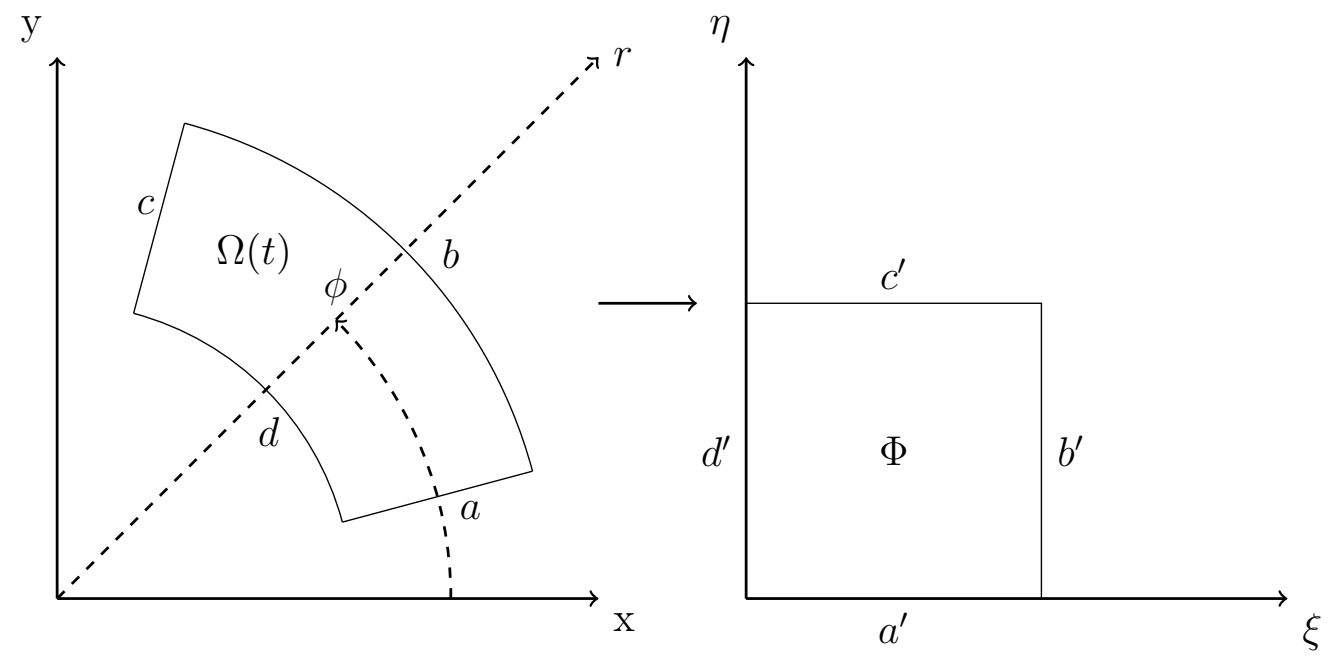

Figure 3: A schematic of the deforming domain

The polar coordinates are

$$
r(x, y, t)=\sqrt{x(t)^{2}+y(t)^{2}}, \quad \phi(x, y, t)=\tan ^{-1}(y(t) / x(t)),
$$

and the exact description of the moving boundaries is given by

$$
\begin{aligned}
& r_{d}(t)=1+0.1 \sin (2 \pi t), \quad r_{b}(t)=2-0.2 \sin (2 \pi t), \\
& \phi_{a}(t)=0+0.1 \sin (2 \pi t), \quad \phi_{c}(t)=(\pi / 2)-0.2 \sin (2 \pi t),
\end{aligned}
$$

where $a, b, c$ and $d$ are shown in Figure 3. Next, we scale the polar coordinates such that the fixed domain becomes the unit square, as

$$
\xi(x, y, t)=\frac{r(x, y, t)-r_{d}(t)}{r_{b}(t)-r_{d}(t)}, \quad \phi(x, y, t)=\frac{\phi(x, y, t)-\phi_{a}(t)}{\phi_{c}(t)-\phi_{a}(t)} .
$$

A schematic of the deforming domain at different times is given in Figure 4.

To determine the order of accuracy of approximation, the method of manufactured solution with

$$
U_{\infty}=\left[\sin \left(x^{2}-t\right), \cos (x-t), \sin (y-t)\right]^{T},
$$

is used. In (70), $\mathbf{U}_{\infty}=\left[U_{\infty}^{T}, V_{\infty}^{T}, W_{\infty}^{T}\right]^{T}, V_{\infty}=\partial U_{\infty} / \partial x$ and $W_{\infty}=\partial U_{\infty} / \partial y$. The error is quantified as $e=U_{\infty}-\tilde{U}$. 


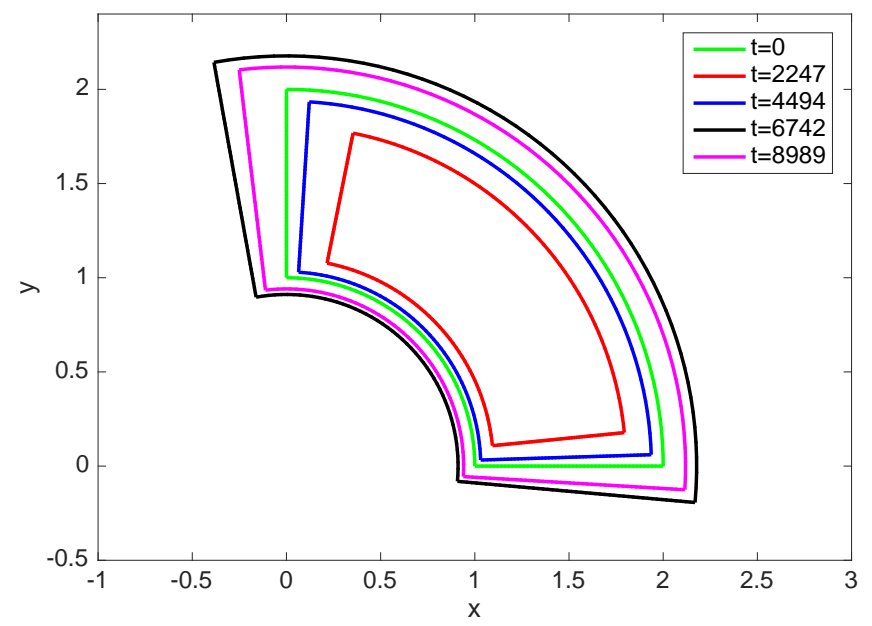

Figure 4: A schematic of the deforming domain at different times

\subsection{Remarks on implementation}

To compute $\tilde{U}$, we rewrite (40) as

$$
\mathcal{L} \tilde{U}=\mathcal{R} .
$$

In $(71), \mathcal{L}$ is a matrix of size $9(K+1)(N+1)(M+1)$ containing all SBP-SAT contributions excluding data. Additionally, $\mathcal{R}$ is a $9(K+1)(N+1)(M+1)$ vector which includes data. We have used the Generalized Minimal Residual Method (GMRES) to solve (71).

\subsection{Order of accuracy for the solution and the convergence}

We examine the scheme for SBP operators of order $2 s$ in the interior and $s$ close to the boundaries, where $s \in\{1,2,3\}$. The fifth order accurate SBP operator SBP84, with a sufficiently large $K$, is used in time.

The rates of convergence are calculated as

$$
p=\frac{\log \frac{\left\|e^{(1)}\right\|_{\bar{J} \mathcal{P}}}{\left\|e^{(2)}\right\|_{\bar{J} \mathcal{P}}} .}{\log \frac{N^{(1)} M^{(1)}}{N^{(2)} M^{(2)}}}
$$

where superscripts (1) and (2) denote two mesh levels with $\left(N^{(1)}+1\right) \times$ $\left(M^{(1)}+1\right)$ and $\left(N^{(2)}+1\right) \times\left(M^{(2)}+1\right)$ grid points, respectively.

In Table 1, the convergence rates of the solution are shown for a sequence of spatial mesh refinements. The results corroborate that the scheme is design order accurate $[1,14]$. 


\begin{tabular}{|c|c|c|c|c|}
\hline$N, M$ & 21 & 31 & 41 & 51 \\
\hline$S B P 21$ & 2.0152 & 1.9970 & 1.9937 & 1.9965 \\
\hline$S B P 42$ & 3.0737 & 3.0012 & 3.0063 & 3.0745 \\
\hline$S B P 63$ & 5.1925 & 3.7985 & 4.2519 & 4.4549 \\
\hline
\end{tabular}

Table 1: Convergence rates for the solution at $\mathrm{T}=1$, for a sequence of mesh refinements, SBP84 in time with sufficiently small time steps is used

\begin{tabular}{|c|c|c|c|c|}
\hline$N, M$ & 21 & 31 & 41 & 51 \\
\hline$S B P 21$ & 1.8270 & 2.1751 & 1.9861 & 2.0334 \\
\hline$S B P 42$ & 9.0553 & 4.3946 & 4.2267 & 4.3441 \\
\hline$S B P 63$ & 15.6189 & 4.9515 & 5.1150 & 4.5048 \\
\hline
\end{tabular}

Table 2: Convergence rates for the divergence at $\mathrm{T}=1$, for a sequence of mesh refinements, SBP84 in time with sufficiently small time steps is used

In Table 2, the convergence rates of the divergence are shown for a sequence of spatial mesh refinements. Due to the first order formulation of the problem, the divergence converges with the design order of accuracy of the scheme.

\subsection{Order of accuracy for functionals}

Based on the theory, a superconverging functional should be achieved for linear problems and linear integral functionals $[6,7,8,9,10]$ for dual consistent approximations. Here we will compute both linear and non-linear functionals to see whether or not superconvergence is obtained also in the nonlinear case.

The linear and non-linear functionals that we consider are

$$
J_{1}(U)=\int_{\Phi} u d \Phi \text { and } J_{2}(U)=\int_{\Phi} \frac{1}{2}\left(u^{2}+v^{2}\right) d \Phi,
$$

respectively. Additionally, we calculate the integral of the divergence as

$$
J_{3}(U)=\int_{\Phi} u_{x}+v_{y} d \Phi .
$$

The exact functionals are computed using (70) and the rates of convergence 


\begin{tabular}{|c|c|c|c|c|}
\hline$N, M$ & 21 & 31 & 41 & 51 \\
\hline SBP21 & 1.9981 & 1.9604 & 1.9756 & 1.9647 \\
\hline SBP42 & 4.0287 & 4.3275 & 4.6774 & 6.0737 \\
\hline SBP 63 & 9.6653 & 6.8172 & 8.8344 & 6.2045 \\
\hline
\end{tabular}

Table 3: Convergence rates for $J_{1}$ at $\mathrm{T}=1$, for a sequence of mesh refinements, $\mathrm{SBP} 84$ in time with sufficiently small time steps is used

\begin{tabular}{|c|c|c|c|c|}
\hline$N, M$ & 21 & 31 & 41 & 51 \\
\hline SBP21 & 1.9933 & 1.9759 & 1.9786 & 1.9815 \\
\hline SBP42 & 3.5550 & 4.0678 & 4.6536 & 5.6233 \\
\hline SBP 63 & 9.5206 & 6.7957 & 2.5920 & 7.4805 \\
\hline
\end{tabular}

Table 4: Convergence rates for $J_{2}$ at $\mathrm{T}=1$, for a sequence of mesh refinements, SBP84 in time with sufficiently small time steps is used

of the numerical functionals toward the exact ones are calculated by using

$$
p=\frac{\log \frac{\left|e^{(1)}\right|_{\bar{J} \mathcal{P}}}{\left|e^{(2)}\right|_{\bar{J} \mathcal{P}}}}{\log \frac{N^{(1)} M^{(1)}}{N^{(2)} M^{(2)}}}
$$

The rates of convergence for SBP21, SBP42 and SBP63 are given in Tables 3-5.

As shown in Tables 3-4, superconvergence is achieved for $J_{1}$ and $J_{2}$. Superconvergence is achieved for $J_{3}$ when using $S B P 21$ and $S B P 42$, but for some reason not for $S B P 63$, as seen in Table 5 .

\begin{tabular}{|c|c|c|c|c|}
\hline$N, M$ & 21 & 31 & 41 & 51 \\
\hline$S B P 21$ & 2.1527 & 2.5231 & 2.5231 & 2.4508 \\
\hline$S B P 42$ & 9.5427 & 4.8729 & 4.9226 & 5.2762 \\
\hline$S B P 63$ & 21.1193 & 1.4537 & 4.3645 & 2.7469 \\
\hline
\end{tabular}

Table 5: Convergence rates for $J_{3}$ at $\mathrm{T}=1$, for a sequence of mesh refinements, SBP84 in time with sufficiently small time steps is used 


\section{Summary and conclusion}

A high order, fully discrete and stable approximation of the linearized incompressible Navier-Stokes on first order form was developed.

The derivations for the continuous problem was done by reducing the second order system to first order form. Boundary conditions that simultaneously lead to well-posedness of the primal and dual problems posed on time-dependent spatial domains were derived.

Stability and dual consistency was obtained by using summation-by-parts operators in combination with the simultaneous approximation term technique. Penalty formulations that adjust to the time variations of the spatial geometry such that stability and dual consistency follow automatically, were derived.

The order of accuracy of the solution, the divergence and linear and non-linear functionals were examined numerically. Design order of accuracy was obtained for the solution and the divergence. The linear and nonlinear functionals superconverged.

\section{References}

[1] B., Strand, Summation by parts for finite difference approximations of d/dx, Journal of Computational Physics, 110:47-67 (1994).

[2] B. Gustafsson, The convergence rate for difference approximations to general mixed initial boundary value problems, Mathematics of Computations, 29(130):396406 (1975).

[3] B. Gustafsson, The convergence rate for difference approximations to mixed initial boundary value problems, SIAM Journal of Numerical Analysis, 18(2):179190 (1981).

[4] S. Nikkar and J. Nordström, Fully discrete energy stable high order finite difference methods for hyperbolic problems in deforming domains. Journal of Computational Physics, 291(0):82-98 (2015).

[5] S. Nikkar and J. Nordström, Energy stable high order finite difference methods for hyperbolic equations in moving coordinate systems, 21 st AIAA Computational Fluid Dynamics Conference, June 24-27 (2013). 
[6] J. Berg and J. Nordström, Superconvergent Functional Output for Timedependent problems using finite differences on summation-by-parts form, Journal of Computational Physics, 231:6846-6860 (2012).

[7] J. Berg and J. Nordström, On the impact of boundary conditions on dual consistent finite difference discretizations, Journal of Computational Physics, 236:41-55 (2013).

[8] J. Berg and J. Nordström, Duality based boundary conditions and dual consistent finite difference discretizations of the Navier-Stokes and Euler equations, Journal of Computational Physics, 259:135-153 (2014).

[9] J. E. Hicken, and D. W. Zingg, The role of dual consistency in functional accuracy: error estimation and superconvergence, 20th AIAA Computational Fluid Dynamics Conference (2011).

[10] J. E. Hicken, and D. W. Zingg, Dual consistency and functional accuracy: a finite-difference perspective, Journal of Computational Physics, 256:161-182 (2014).

[11] C. Farhat, P. Geuzaine, C. Grandmont, The discrete geometric conservation law and the nonlinear stability of ALE schemes for the solution of flow problems on moving grids, Journal of Computational Physics, 174: 669-694 (2001).

[12] J. Nordström, The use of characteristic boundary conditions for the Navier- Stokes equations, Computers \&f Fluids, 24(5):609-623 (1995).

[13] J. Nordström, Conservative finite difference formulations, variable coefficients, energy estimates and artificial dissipation, Journal of Scientific Computing, 29, (2006).

[14] M. Svärd and J. Nordström, On the order of accuracy for difference approximations of initial boundary value problems, Journal of Computational Physics, 218:333-352 (2006).

[15] Saul S. Abarbanel and Alina E. Chertock, Strict stability of highorder compact implicit finite-difference schemes: the role of boundary conditions for hyperbolic PDEs, I, Journal of Computational Physics, 160(1):42-66 (2000). 
[16] Saul S. Abarbanel and Alina E. Chertock and Amir Yefet, Strict stability of high-order compact implicit finite-difference schemes: the role of boundary conditions for hyperbolic PDEs, II, Journal of Computational Physics, 160:67-87 (2000).

[17] Mark H. Carpenter and David Gottlieb, Spectral methods on arbitrary grids, Journal of Computational Physics, 129:74-86, (1996).

[18] David C. Del Rey Fernández and Pieter D. Boom and David W. Zingg, A generalized framework for nodal first derivative summation-by-parts operators, Journal of Computational Physics, 266:214-239 (2014).

[19] J. Nordström and T. Lundquist, Summation-by-parts in time, Journal of Computational Physics, 251:487-499 (2013).

[20] T. Lundquist and J. Nordström, The SBP-SAT technique for initial value problems, Journal of Computational Physics, 270:86-104 (2014).

[21] B. Gustafsson, H.-O. Kreiss, and J. Oliger, Time dependent problems and difference methods, John Wiley \& Sons, New York, 1995.

[22] Charles F. Van Loan, The ubiquitous Kronecker product, Journal of Computational and Applied Mathematics, 123:85-100 (2000).

[23] Thomas, P.D., Lombard, C.K., Geometric conservation law and its application to flow computations on moving grids, AIAA Journal, 17: 10301037 (1979).

[24] C. T. Kelley and David E. Keyes, Convergence analysis of pseudotransient continuation, SIAM Journal of Numerical Analysis, 35(2): 508523 (1998).

[25] A. Jameson, Time dependent calculations using multigrid, with applications to unsteady flows past airfils and wings, AIAA 10th Computational Fluid Dynamics Conference, June 24-26 (1991). 